\title{
Procéduralisation et transformation de l'idée de justice
}

Jean-Marc Sorel"

"Certains pensent que l'honnêteté est toujours la meilleure attitude. C'est une superstition. A certains moments, l'apparence de l'honnêteté surpasse grandement l'honnêteté elle-même. »

Mark Twain - Aphorismes

\section{Introduction}

"Justice must not only be done, but it must also be seen to be done. » Cet adage bien connu sous plusieurs versions (et plusieurs traductions) serait en quelque sorte le creuset de la procéduralisation, ou un de ses aspects. Si la justice est rendue, elle doit l'être dans une apparence de justice, la question centrale étant sans doute lorsque l'apparence supplante le rendu de la justice elle-même, autrement dit lorsque le respect de la forme permet d'oublier l'éventuelle vacuité ou la pauvreté du fond. Il est néanmoins évident que la grande majorité des décisions rendues aujourd'hui par les juridictions internationales allient l'apparence et le rendu d'une décision justifiée au fond. Notre propos n'est donc pas de remettre en cause cette évidence, mais de s'interroger sur une balance qui tend de plus en plus à privilégier le respect de la forme, sans forcément amoindrir le fond, mais, pour le moins, à créer des contraintes qui satisfont l'apparence sans forcément satisfaire toujours la justice.

Larme de la procédure est devenue un enjeu à part entière (et, de plus en plus, un objet d'étude ${ }^{1}$ ), gagner un procès sur des arguments de procédure n'est plus une victoire à la Pyrrhus. A cet égard, et pour reprendre l'interrogation centrale de ce panel, on est en droit de s'interroger sur l'importance prise par la procédure - et jusqu'où - dans le règlement des diffé-

* Professeur à l'Ecole de Droit de la Sorbonne (Université Paris 1).

1 Pour un exemple récent : N. Le Bonniec, La procéduralisation des droits substantiels par la Cour européenne des droits de l'homme, Réflexion sur le contrôle juridictionnel du respect des droits garantis par la Convention européenne des droits de l'homme (2015) [Thèse Montpellier 1, sous la direction de F. Sudre]. 
rends internationaux. Par procéduralisation, il faut entendre la progression des éléments formels de procédure à l'intérieur d'un procès ou d'une juridiction en général, progression qui permet le constat d'une forme de domination de l'utilisation du cadre procédural au regard du traitement du fond de l'affaire. Il doit être clair que cette progression n'est pas a priori négative car elle participe de l'évolution, de la maturation, d'une juridiction et des éléments qui permettent de la faire entrer dans le cadre d'un état de droit. Ceci doit être précisé puisqu'il s'agira ici, a contrario, plutôt de voir les effets négatifs de cette procéduralisation. En soulignant ces aspects cela ne doit pas signifier qu'ils sont uniques, ni même prédominants.

Ceci étant, il ne s'agira nullement, dans ce bref papier, d'une étude de la " procédure » au sens strict, ni de la jurisprudence afférente à la procédure, mais plus d'une modeste réflexion sur ce phénomène. ${ }^{2}$ En effet, traditionnellement, on prend en compte les règles substantielles dans le règlement des différends et on néglige les normes procédurales considérées comme secondaires ou formelles dans la décision finale. En bref, le fond l'emportait sur la forme. Qu'en est-il désormais?

Plusieurs brèves - et modestes - remarques permettent d'essayer de circonscrire ce phénomène de procéduralisation du contentieux qui ne semble pas toucher uniquement les juridictions internationales, mais qui serait le marqueur d'une certaine idée de la justice aujourd'hui très répandue. A cet égard, les juridictions internationales semblent plus s’inscrire

2 Pour cette raison, nous omettrons les notes techniques ou les références à de multiples études sur le sujet. Et nous renvoyons à différentes études qui ont émaillé notre réflexion, et dans lesquels nous piocherons si nécessaire pour les remarques qui suivent. Voir notamment : J. M. Sorel, International Courts and Tribunals, Procedure, Max Planck Encyclopedia of International Law (2007), disponible à l'adresse : http://opil.ouplaw.com/view/10.1093/law:epil/9780199231690/law-97801 99231690-e40? rskey=Q6Tzrl\&result=1\&prd=EPIL (dernière visite le 23 octobre 2018), et version papier (2008), 11; J. M. Sorel, Les premiers pas des juridictions internationales : maladresse, péché de jeunesse ou affirmation ?, in Liber Amicorum Jean-Pierre Cot : Le procès international (2009), 283-308; J. M. Sorel, Le paradigme de la constitutionnalisation vu du droit international : le côté obscur de la force, in S. Hennette-Vauchez \& J. M. Sorel (dir.), Les droits de l'homme ont-ils constitutionnalisé le monde ? - Collection de la Convention européenne des droits de l'homme (2011), 217-238; J. M. Sorel, Chapitre 63 : Les tribunaux mixtes ou hybrides, in $\mathrm{H}$. Ascensio et al. (dir.) Droit international pénal (deuxième édition révisée) (2012), 825-843; J. M. Sorel, Les juridictions internationales, in P. Hassner, Les relations internationales, $2^{\text {ème }}$ éd. (2012), 58-63; J. M. Sorel, La justice pénale : un modèle ou une illusion? in S. Guinchard \& J. Buisson (dir.), Les transformations de la justice pénale, cycle de conférences 2013 à la Cour de cassation (2014), 297-309. 
dans l'air du temps que provoquer ce phénomène. Sans épuiser un vaste sujet qui fait l'objet de l'axe directeur de l'Institut Max Planck du Luxembourg sous la houlette d'Hélène Ruiz Fabri, il convient de remarquer l'importance et la généralisation du mouvement de procéduralisation (I), les mille visages de la procédure selon les acteurs du procès (II), la confusion qui en résulte entre procéduralisation et légitimation (de la juridiction et du procès) (III), avant de conclure sur la transformation progressive de l'idée de justice à travers la procéduralisation (IV).

\section{La lame de fond du mouvement de procéduralisation}

Les causes de ce mouvement de procéduralisation sont multiples et, pour la plupart, bien connues. On pourrait, par simplification excessive, se contenter de remarquer la propagation souvent remarquée de la sphère anglo-saxonne sur les autres systèmes juridiques, et sur les juridictions internationales en particulier. En effet, la procédure y tient une place depuis longtemps importante, pour ne pas dire démesurée. Les juridictions internationales pénales en ont été, à cet égard, des révélateurs par la prédominance du système accusatoire de la common law. Mais sans doute faut-il plus pointer du doigt la progression de ce qu'il est convenu de qualifier de théorie des apparences procédurales. Ce mouvement s'appuie sur une soif de légalisation, de juridisme, qui fait passer la forme avant le fond, et qui modifie souvent le sens du procès. L'espoir ne sera pas de gagner le juge à la cause défendue, mais de le faire trébucher sur des arguments formels. On souhaite que la forme soit respectée, et on s'arcboute sur celle-ci, sans se soucier de savoir si le fond en devient - ou en sera - plus impartialement jugé.

\section{A. Un exemple récent et topique en droit interne français}

On peut en juger dans bien d'autres domaines que les juridictions internationales. Ainsi la bataille des critères d'impartialité devant les autorités administratives indépendantes en France qui fait rage depuis quelques années nous semble un parfait exemple qui a récemment rebondi. En effet, une ju- 
risprudence récente du Conseil d'Etat français peut à cet égard illustrer cette dérive procédurale. ${ }^{3}$

En effet, le Conseil d'État vient d'accepter d'être saisi de recours en annulation contre des actes de droit souple, tels que des communiqués de presse ou des prises de position d'autorités publiques, alors que de tels actes n'étaient jusqu'alors pas susceptibles de recours juridictionnels dès lors qu'ils n'avaient aucun effet juridique (nous préférerions préciser " aucun effet obligatoire»). Petite révolution donc dans le droit français. Mais quelle révolution?

Les deux affaires jugées par l'assemblée du contentieux montraient l'importance du droit souple dans les nouveaux modes d'action des personnes publiques, comme l'avait souligné l'étude annuelle du Conseil d'État de l'année 2013 consacré au "Droit souple ». Sans véritablement créer d'obligation juridique ni accorder de nouveaux droits aux usagers, l'administration peut utiliser des instruments de communication pour influencer ou dissuader les acteurs, et peut émettre des prises de position ou des recommandations qui n'ont pas de valeur obligatoire mais vont, dans les faits, être écoutées et suivies d'effets. A ce propos, le Conseil d'Etat juge tout d'abord, conformément à une jurisprudence antérieure, qu'il peut être saisi lorsqu'il s'agit d'avis, de recommandations, de mises en garde et de prises de position qui pourraient ensuite justifier des sanctions de la part des autorités. Ensuite, et sur ce point de manière novatrice, il se reconnait compétent lorsque l'acte contesté est de nature à produire des effets notables, notamment de nature économique, ou lorsqu'il a pour objet d'influer de manière significative sur les comportements des personnes auxquelles il s'adresse. Pour examiner la légalité de ces actes, le juge contrôle, en l'espèce, la compétence des personnes publiques pour les édicter, le respect des droits de la défense et, avec une intensité variable selon les actes en cause, l'appréciation portée par l'autorité. Mais c'est bien le respect du cadre procédural qui semble l'épicentre de cette petite révolution en droit français.

9. [...] qu'il ressort ainsi des pièces du dossier que la société $\mathrm{NC} \mathrm{Nu-}$ mericable a pu présenter ses observations préalablement à l'adoption de l'acte attaqué; que, par suite, le moyen tiré de ce que l'Autorité aurait méconnu le principe général des droits de la défense, au motif

3 Société Fairvesta International GMBH et autres, décisions $\mathrm{n}^{\circ} 368082,368083$, 368084 : CE, 21 mars 2016; Société NC Numericable,décision n 390023 : CE, 21 mars 2016. 
qu'elle n'aurait pas consulté la société NC Numericable préalablement à l'adoption de sa délibération, doit être écarté;

10. Considérant, d'autre part, que l'acte attaqué a été, comme il a été dit ci-dessus, délibéré par la commission permanente de l'Autorité de la concurrence; que la lettre du président adressée à la société requérante a pour objet de lui notifier cet acte et de lui en donner les motifs; que, par suite, les moyens tirés de l'incompétence du président de l'Autorité pour prendre un tel acte, de ce qu'il ne procéderait pas d'une délibération collégiale et qu'il serait entaché d'irrégularité en ce qu'il méconnaîtrait le principe de parallélisme des formes et des procédures, ainsi que le principe de parallélisme des compétences, doivent être écartés. ${ }^{4}$

On observe dans ce domaine, comme dans d'autres, un glissement entre le contrôle substantiel et le contrôle procédural en matière économique, ce qui conduit finalement à préserver une marge de manœuvre aux autorités administratives chargées de réguler des secteurs économiques quant au contenu des choix effectués, tant que ceux-ci sont effectués et régulés conformément à la loi. En réalité, la validité axiologique des choix effectués par le juge, c'est-à-dire la conformité du produit de l'interprétation à des valeurs exogènes, n'a que guère d'importance car il est probable, sauf " erreur manifeste d'appréciation » (elle-même peu probable), que l'interprétation au fond sera conforme à celle édictée par l'autorité professionnelle régulatrice. On peut par ailleurs s'en féliciter pour l'essence même de la régulation économique. Tout au plus, cela peut être un signal vers l'attention qui doit être portée aux avis ou textes souples en cause. La prudence sera de mise dans leur rédaction. L'essentiel résidait bien dans l'apparence du contrôle juridictionnel effectué sur des textes souples, et dans le contrôle avant tout du respect d'une procédure permettant de les invoquer.

Mais il en va de même, plus prosaiquement, pour certaines procédures non juridictionnelles. L'universitaire français ne peut que remarquer - et souvent regretter - que les procédures de recrutement au sein de l'université, notamment pour les professeurs, s'assimilent à une véritable parodie procédurale : l'administration va se soucier de vérifier le nombre de jours entre deux réunions, le quorum, la parité au sein des comités, etc, sans se soucier par ailleurs de savoir si le candidat (bien souvent préprogrammé et unique) a vraiment été choisi sur des critères d'excellence. Peu importe

4 Société NC Numericable, supra note 3. 
donc si la procédure ne sert qu'à avaliser un candidat pressenti d'une manière absolument pas démocratique. L'apparition du rapprochement de conjoints dans ce cadre permet même désormais de se passer de toute appréciation sur le fond du dossier. Les Universités, effrayées par une menace de recours n'osent plus bouger le petit doigt. C'est dire.

Tout est contestable et souvent contesté formellement. Dès lors, on expose tout, sans pour autant que le « rendre compte " (accountabilty) modifie le fond de la question. Ce qui compte, c'est le respect de la forme, et peu importe si le fond est toujours aussi subjectif, voire totalement partial.

\section{B. La procéduralisation devant les juridictions internationales}

Si nous revenons aux juridictions internationales, partant du constat bien connu que le fondement de la juridiction internationale serait le consentement de l'Etat sur la base de la matrice de la Cour internationale de Justice, on incline rapidement à penser que la procédure est le rempart derrière lequel l'Etat va s'abriter pour que sa volonté soit scrupuleusement respectée. Ce qui revient à admettre que cette procédure est avant tout une forme de droits de la défense - et non d'expression de droits positifs - pour empêcher la frontière bien gardée du consentement d'être contournée. Certes, ceci est logique pour le défendeur devant toute juridiction, mais elle prend ici une dimension particulière puisqu'il faut prima facie constater que le justiciable a bien accepté son juge. Cette posture reste fondamentalement vraie en dépit de la progression de formes de juridiction obligatoire en droit international. Certes, le "monolithe s'effrite ${ }^{5}$ mais la place de la Cour internationale de Justice reste centrale, voire supérieure par son ancienneté et son aura, à défaut de posséder une position suprême. A bien des égards donc, la CIJ peut servir de matrice à une réflexion sur le mouvement de procéduralisation.

Pour les Etats, la juridiction consentie est une protection et l'on a vu la CIJ s'épuiser dans de longs procès intermédiaires pour prouver ou rejeter sa compétence, ou la recevabilité de la requête. Parfois avec suspicion lorsque ce refus aurait pu être indiqué dès le stade des exceptions préliminaires

5 H. Ruiz Fabri \& J. M. Sorel, Juridiction obligatoire ? Procédure contraignante ? Et si l'amoindrissement de la liberté des Etats face à leurs juges ne venait pas d'où l'on pense ?, in Justices et droit du procès, du légalisme procédural à l'humanisme processuel, Mélanges en l'honneur de Serge Guinchard (2010), 479-490. 
(Sud-Ouest africain ${ }^{6}$ ou Barcelona Traction ${ }^{7}$ ) mais, quoi qu'il en soit, les arrêts sur la compétence ou la recevabilité ont toujours été considérés comme au moins aussi importants que ceux sur le fond parce qu'ils révèlent des possibilités ou limites de la juridiction internationale.

On le sait, la CIJ fonctionne sur la base d'un statut ancien et peu renouvelé, auquel s’adjoint un Règlement de procédure qui est lui évolutif, ne serait-ce que parce que le juge en a cette fois-ci la maîtrise. Les instructions de procédure venant compléter cet ensemble. Il n'empêche qu'une disproportion entre le Statut et le Règlement s'est faite jour rapidement. Les traces de dispositions procédurales sont souvent ténues dans le Statut, alors que le Règlement peut lui accorder toute leur importance. Quoi qu'il en soit, l'équilibre n'est plus respecté entre les quelques allusions statutaires à certaines procédures, et leur utilisation par les Etats. La liaison entre le Statut et le Règlement pourrait être largement améliorée si toutes les procédures y trouvaient une place, et si l'équilibre entre les procédures était respecté dans la rédaction des articles. ${ }^{8}$ Mais un autre constat peut être opéré. La progression de la juridiction obligatoire entraîne aussi ses effets pervers dont la montée en puissance de la procéduralisation pourrait être un signe. Ce serait, en quelque sorte, le prix à payer pour amoindrir le passage du consentement à l'obligatoire pour les Etats.

Une distinction historique est nécessaire entre la CIJ qui a toujours privilégié la procédure en raison de la sensibilité historique des Etats souverains dans le cadre d'une bonne administration de la justice et d'une justice consentie (mais sans l'obsession de l'administration de la preuve qu'elle laisse venir), alors que les nouvelles juridictions privilégient la procédure pour d'autres raisons : prouver que l'on est sérieux, assurer les premiers pas d'une juridiction, s'affirmer face à l'Etat, etc. Le syndrome du débutant en quelque sorte. Mais, en général, pour des raisons différentes, toutes les juridictions sont touchées par ce mouvement qui semble venir d'une forme de

6 Affaires du Sud-Ouest africain (Éthiopie c. Afrique du Sud; Libéria c. Afrique du Sud), Exceptions préliminaires, Arrêt, CIJ Recueil 1962, 319; Affaires du Sud-Ouest africain (Éthiopie c. Afrique du Sud; Libéria c. Afrique du Sud), Deuxième phase, Arrêt, CIJ Recueil 1966, 6.

7 Affaire de la Barcelona Traction, Light and Power Company, Limited (Belgique c. Espagne), Mémoires, Plaidoiries et Documents, CIJ Recueil 1962, 2; Affaire de la Barcelona Traction, Light and Power Company, Limited (Belgique c. Espagne), Arrêt, CIJ Recueil 1970, 3.

8 J. M. Sorel \& F. Poirat, Rapport introductif : Les procédures incidentes devant la Cour internationale de Justice : exercice ou abus de droits?, Université de Rennes 1 - Journée d'études du 18 mai 2000 : Collection contentieux international (2001), 7, 7-57. 
principe général $d e$ droit. Et, à cet égard, le principe général $d u$ droit selon lequel la juridiction en droit international est consentie parce que, justement, il ne peut être tiré d'un principe général de droit en sens inverse, semble dangereusement vaciller. Le principe se dilue, oscille entre le «commun » et le "général », entre le «de » et le «du», sans que l'on sache désormais dans quel sens la balance penche.

Le progrès de la juridiction obligatoire - donc de la juridictionnalisation du droit international - est, on le sait, à la fois quantitatif et qualitatif. Or, dans ce qualitatif, la procédure a un rôle important dont la justice internationale pénale a montré à bien des égards la richesse et les effets pervers. Larrivée de la procédure pénale en droit international a clairement entraîné une sensibilité accrue aux exigences du procès équitable, même si la propension au contradictoire et à l'égalité des armes était déjà dans les gênes du procès interétatique, confronté à la susceptibilité des clients étatiques. Mais, surtout, ceci a créé une sorte de mouvement de propagation de la sensibilité aux aspects procéduraux, d'autant qu'il existe une claire tendance des juridictions à s'inspirer de ce qui se fait ailleurs pour résoudre les questions procédurales qui pourraient surgir devant elles. Le phénomène fut particulièrement prégnant pour la justice pénale qui, privée de modèle original en droit international, s'est moulée dans les modèles nationaux, parfois à l'excès comme le TPIY pour le modèle anglo-saxon. Mais ce phénomène qui, à l'origine, partait de l'interne vers l'externe, semble désormais subir une influence horizontale entre les juridictions internationales. "Ce fut tout aussi prégnant pour un mécanisme jeune comme le règlement des différends de l'OMC, qui s'est explicitement référé à la jurisprudence d'autres cours et tribunaux pour fonder, et en même temps, légitimer ses solutions, alors même que la procédure était déjà, de façon générale, le domaine où l'on rencontrait les exemples les plus évidents de principes généraux du droit international. De fait, c'est bien ce phénomène qu'on observe, de généralisation de principes de procédure. La convergence ou l'entrelacement de ces deux facteurs favorise une homogénéisation, qui est aussi un facteur de contrainte pour les justiciables mais qui est en même temps, et cela peut rencontrer leur revendication, un facteur de sécurité ».9

On le conçoit aisément, le débat n'est pas simple et le miroir de la procéduralisation est à multiples facettes. On constate ainsi que la progression de la juridiction obligatoire en droit international doit sans doute plus à une procéduralisation contraignante qu'à la progression proprement dite

9 Ruiz Fabri \& Sorel, supra note 5, 488. 
du consentement des Etats. Le tout s'ajuste dans un système de vases communicants : la procédure s'affirme par banalisation mais aussi comme bouclier contre l'effritement du facultatif. La procéduralisation apparait ainsi comme une cause de la progression du caractère obligatoire de la juridiction internationale, mais aussi comme une conséquence de celle-ci. Etant de plus en plus exposé, on se protège de plus en plus.

De leur côté, les juridictions internationales, faute d'un ancrage matriciel dans la sphère internationale, faute d'être le point d'équilibre initial entre des pouvoirs constitués, faute d'un pouvoir judiciaire unifié, doivent trouver en elles-mêmes la source de leur autorité et de leur légitimité.

\section{Les mille visages de la procédure selon les acteurs du procès}

La procédure peut être envisagée différemment selon les acteurs d'un procès international : C'est un Janus aux mille visages. Mais, finalement, tout le monde peut y trouver un intérêt, sauf peut-être la justice elle-même.

Il existe une différence essentielle entre le client qui, lorsqu'il s'agit d'un Etat, comprend souvent mal les règles de procédure et les estime superfétatoires, et les Conseils dont la mission est notamment de rappeler au client toute l'importance des arguties de procédure. Quant au juge, on pourrait résumer sa philosophie par une forme d'aphorisme : l'utilisation des règles procédurales n'est pas forcément souhaitée (sous-entendue, lorsqu'elles le sont avec excès), mais c'est permis, et donc encore moins interdit.

Si la volonté des Etats est encore effective au stade de l'acceptation de la juridiction, elle s'amoindrit dès lors qu'un procès se déroule dans lequel ils sont impliqués. Une fois dans la nasse, on est cerné par la procédure. Alors que la cause paraissait claire pour l'Etat, il s'inquiète souvent des détours de la procédure qui sont, pour lui, autant d'obstacles inutiles vers l'affirmation de sa thèse. A l'inverse, il peut s'en trouver réconforter si, en tant que défendeur, il est conscient que le jugement au fond lui sera probablement défavorable. C'est alors au Conseil d'utiliser au mieux cette procédure, que ce soit dans un sens ou dans l'autre.

Qu'on nous permette l'évocation d'une expérience personnelle comme Conseil et avocat du Cambodge dans l'affaire de la Demande en interprétation de l'arrêt du 15 juin 1962 en l'affaire du Temple de Préab Vihéar (Cambodge c. Thaillande), devant la Cour internationale de Justice (arrêt du 11 
novembre 2013). ${ }^{10} \mathrm{Il}$ y avait alors remise en cause de la procédure de 1962 et utilisation de l'actuelle, mais surtout cette procédure s'est révélée un piège pour la Thailande 50 ans après l'arrêt initial. En l'espèce, le juge a joué l'application à la lettre de la procédure en répondant à une situation inédite : une demande en interprétation d'un arrêt rendu 50 ans plus tôt. Estimant que la Thaillande avait donné son consentement à l'affaire initiale (en l'espèce, elle avait alors fait la déclaration facultative de juridiction obligatoire), la Cour, se basant sur la seule interprétation de l'arrêt initial, a estimé que ce consentement était toujours valable, alors même que la Thailande n'avait plus de déclaration de l'article 36 para. 2. Au surplus, des mesures conservatoires ont été demandées par le Cambodge (et acceptées par la Cour) après l'introduction de la demande en interprétation. Certes, il ne s'agissait pas d'une première puisque le Mexique avait fait de même en 2009 mais, alors que le Mexique avait introduit une affaire en interprétation au principal pour demander des mesures conservatoires (car il s'agissait alors de l'unique possibilité), son objectif étant uniquement l'obtention de ces dernières, ${ }^{11}$ le Cambodge avait bien comme finalité l'interprétation de l'arrêt de 1962, ce à quoi la Cour a fait droit en interprétant réellement pour la première fois un de ses arrêts avec des conséquences bien concrètes pour les parties.

Si, dans le cas du Mexique, la procédure lui a permis d'atteindre son but (obtenir des mesures conservatoires), tout en voyant logiquement rejeté sa demande au fond, pour le Cambodge, l'utilisation de la procédure n'avait pour objectif que de passer l'obstacle de manière à parvenir au fond. Quoi qu'il en soit, le maniement des possibilités ouvertes par la procédure fut remarquable, ouvrant des perspectives et une souplesse qui n'étaient pas évidentes à la simple lecture du Statut ou même du Règlement. Dans ce cadre, le Conseil de l'Etat se doit d'envisager les possibilités, tout en expliquant à l'Etat les risques de l'utilisation de certaines procédures. Ceci fonctionne comme indiqué supra par défaut : si ce n'est pas interdit, c'est que c'est possible. Encore faut-il bien utiliser cette procédure et envisager toutes les situations où la Cour pourrait, par une ouverture insoupçonnée, rejeter la demande. A l'inverse, lorsque l'affaire est amenée par compromis, le juge se concentre directement sur le fond, ce qui se révèle plus efficace et ren-

10 Affaire du temple de Préah Vihéar (Cambodge c. Thailande), Arrêt, CIJ Recueil $1962,6$.

11 Demande en interprétation de l'arrêt du 31 mars 2004 en l'affaire Avena et autres ressortissants mexicains (Mexique c. Etats-Unis d'Amérique) (Mexique c. EtatsUnis d'Amérique), Arrêt, CIJ Recueil 2009, 3. 
voie la procédure à ce qu'elle est : une arme aux mains des parties pour empêcher ou retarder le dénouement sur le fond.

Cette évolution ne joue pas seulement dans le domaine du contentieux puisque l'on constate également une forme de contentieurisation (on nous pardonnera ce barbarisme) de la procédure consultative. Entre autres exemples, l'affaire dite du Mur en Cisjordanie en 2004 en a été l'illustration. ${ }^{12}$ La Cour, avant de répondre à la question d'une manière ferme, a été dans l'obligation de se débarrasser de nombreuses exceptions opposées à sa compétence ou à la recevabilité de la question. Est-ce la raison pour laquelle, elle a elle-même dépassé le cadre de l'avis consultatif en posant des injonctions en termes de responsabilité aux Etats qui favoriseraient la perpétuation de ce mur?

Alors même que les Etats ont (re)découvert les biais de procédure qui s'ouvrent à eux, ce décalage pose problème. On songe par exemple au Nigéria qui, dans l'Affaire de la frontière terrestre et maritime ${ }^{13}$ qui l'opposait au Cameroun a utilisé toute la gamme des procédures incidentes (mesures conservatoires, exceptions préliminaires, interprétation de l'arrêt sur les exceptions préliminaires, intervention, demande reconventionnelle) pour retarder l'inéluctable échéance. Si l'attitude dilatoire ne trompait personne, la Cour a joué le jeu, tout en signifiant dans ses réponses (parfois très courtes) le caractère inapproprié de la demande (par exemple, en répondant par quelques lignes sibyllines à la demande reconventionnelle dans l'arrêt au fond). La Cour n'est donc pas démunie devant l'abus de procédure mais elle ne peut que «signifier » son irritation d'une manière indirecte, à défaut de l'exprimer en la contrecarrant ouvertement.

L'attitude des juges vis-à-vis de ces tendances est donc ambivalente. Ils la provoquent parfois par leur attitude, parfois ils en sont le réceptacle obligé. Finalement, ils ont peu de marge de manœuvre en eux-mêmes même s'ils peuvent apprécier différemment les procédures. Et, comme souvent, ils s'en serviront en fonction de ce qu'ils souhaitent répondre sur le fond. A cet égard, on ne peut oublier que la procédure peut être une arme utile au juge qui ne souhaite pas s'encombrer d'une affaire délicate à trancher au fond.

Pour le juge international, il existe donc une claire hésitation concernant la question de l'abus de procédure. Non que la procédure ne soit par-

12 Conséquences juridiques de l'édification d'un mur dans le territoire palestinien occupé, Avis Consultatif, CIJ Recueil 2004, 136.

13 Affaire de la Frontière terrestre et maritime entre le Cameroun et le Nigéria (Cameroun c. Nigéria; Guinée équatoriale (intervenant)), Arrêt, CIJ Recueil 2002, 303. 
fois prévue. Ainsi, il est possible de se référer à l'article 294 para. 1 de la Convention de Montego Bay sur le droit de la mer (Partie XV, Règlement des différends) qui indique, concernant les procédures préliminaires, que la Cour ou le Tribunal peut décider si une demande est un « abus des voies de droit ", à l'image des juridictions internes. Si la réponse est positive, il cesse d'examiner la demande. La solution est sans doute radicale et peutêtre guère praticable, mais elle a le mérite de mettre l'accent sur une question trop souvent tabou, et peut-être d'éviter de subir ces abus comme l'exemple de l'affaire entre le Cameroun et le Nigéria l'a prouvé.

Mais le juge n'est pas qu'un spectateur impuissant face à la procédure initiée par les parties. En effet, il n'est que de rappeler que plus on donne à un juge l'occasion de se prononcer, plus on lui donne la possibilité de consolider la procédure et de s'affranchir de la volonté des parties. Au surplus, sa compétence d'auto-règlementation lui ouvre toujours la possibilité d'infléchir certaines procédures, de les développer ou de les freiner selon ce qu'il souhaite en faire, et que les parties en fassent.

Finalement, la procéduralisation est la rencontre entre le souhait des parties et ceux du juge. Même là où la contrainte procédurale n'existe pas en principe, on a de plus en plus tendance à vouloir l'instaurer, car elle est ressentie comme facteur de sécurité. Ceci signifie que le juge se protège par la procédure autant qu'il pense protéger les parties en s'inscrivant dans la durée par une procédure établie et, si possible, stable. Et ceci joue dans toutes les sphères des juridictions internationales, y compris dans l'arbitrage. Incontestablement, il y a une procéduralisation du processus arbitral, tout comme il y a une forme d'arbitralisation de la procédure juridictionnelle (notamment, mais pas seulement, à travers le phénomène des chambres restreintes ouvertes dans de nombreuses juridictions internationales).

La procédure est donc envisagée différemment selon les acteurs d'un procès international. Elle représente une arme (défensive et offensive) pour les parties, elle représente une affirmation et une légitimation pour le juge, mais aussi une défense selon qu'il adopte l'attitude d'un juge spectateur, d'un juge utilisateur de cette procédure comme moyen de défense contre des parties trop entreprenantes, ou d'un simple juge "sécurisant ». Dès lors, on le conçoit aisément, l'arme procédurale est à multiples tranchants pour les acteurs du procès, et aucune simplification excessive de sa vision ne peut convenir. 


\section{La confusion entre la procéduralisation et la légitimation}

La question la plus délicate, et celle pour laquelle aucune réponse péremptoire n'existe, est celle de la liaison entre la procéduralisation et la légitimation des juridictions. En simplifiant, l'impression est que tout renforcement procédural peut être assimilé à un renforcement de la juridiction, et à sa légitimation, entendu comme un processus qui permet à cette juridiction de s'affirmer en tant qu'organe tiers au regard de ses propres justiciables.

Quoiqu'on en dise, les juridictions internationales sont à la recherche d'une affirmation et d'une légitimation. Elles sont souvent fragiles et doivent se consolider pour affronter des justiciables pas tout à fait comme les autres, que ce soient les Etats, les organisations internationales ou les personnes privées alors dans une situation bien particulière. Dès lors, la liaison entre la procéduralisation et la légitimation s'impose. Elle s'impose à la justice internationale permanente, elle s'est imposée au début de la justice internationale pénale construite sur des soubassements fragiles, et elle s'impose de plus en plus - certes dans une moindre mesure - à la justice commerciale de l'OMC.

En effet, la procédure est le ciment qui reste quand il y a-comme ce fut le cas au tournant des XXe et XXIe siècles - des juridictions qui apparaissent d'une manière difficile à appréhender : justice pénale (sous trois formes différentes : ad hoc, permanente (CPI) ou internationalisée), commerciale (OMC), en matière de droit de la mer (TIDM plus proche du modèle de la CIJ), arbitrages (CIRDI ou de nouveau le droit de la mer). Consécutivement, l'interétatique se noient dans un ensemble plus vaste avec une variété de justiciables, de types de procès (civil - mutatis mutandis - pénal, commercial, etc.). Y accoler une procédure rigide devient la garantie de son sérieux, mais surtout la source de sa légitimité dans un univers qui ne connait pas de code de procédure unifié, où tout reste à construire, à imposer. Contrairement à la justice interne, le temps de maturation ne peut qu'être court et est soumis à une perpétuelle médiatisation.

Même si le débat peut paraître galvaudé, la liaison entre procéduralisation et légitimation est essentielle car, dans ce domaine, les produits du droit international veulent ressembler aux Etats. On connait le danger de l'étato-morphisme, mais il faut reconnaître que le modèle interne imprègne encore largement des juridictions qui, faute d'un pouvoir judiciaire unifié, doivent trouver en elles-mêmes la source de leur autorité et de leur légitimité qui ne leur est pas donnée de l'extérieur.

C'est en effet la procédure qui rend légitime et qui va renforcer la juridiction. A défaut, elle paraîtra rendre une justice expéditive et sans fonde- 
ments. Ce hiatus est parfois présent quand une juridiction se saisit d'une question dont elle ne peut que difficilement traiter pour des raisons avant tout procédurales. Ainsi dans l'affaire dite du génocide entre la Bosnie et la Serbie devant la CIJ en 2007, ce fut bien la délicate frontière entre le modèle procédural pénal et le modèle procédural pour la responsabilité internationale qui s'avérait fondamentale dans cet arrêt. ${ }^{14}$

Le ballet procédural de la charge de la preuve en fut le symptôme. Ici, le défendeur niait et n'apportait pas de preuves contraires, et le demandeur était (quasiment) laissé à sa solitude. En ce sens, la Cour internationale de Justice a prouvé qu'elle n'était pas adaptée au procès pénal que contraint l'étude du génocide. ${ }^{15}$ In fine, c'est la remise en cause d'un modèle juridictionnel au regard d'un certain type de procès qui doit retenir l'attention. L'inadaptation de la Cour à un procès de type pénal surgit alors qu'on lui demande de ne pas juger pénalement parce que ce n'est pas son rôle, et que l'Etat ne peut être pénalement responsable, tout en la priant de prendre position sur une incrimination qui ressort clairement du domaine pénal. Difficile d'affirmer sans doute que la Cour internationale de Justice n'était pas légitime parce qu'inadaptée à ce type de procès, mais le sentiment qu'elle n'était guère à sa place au cour de cette procédure a été dans beaucoup d'esprits.

Quoi qu'il en soit, la procédure participe à la juridictionnalisation car, avec un " processus procédural de plus en plus contraignant et stéréotypé, on aboutit à un filet dont les mailles sont de plus en plus resserrées ${ }^{16}$ D'autant qu'une forte pression sociale joue en faveur d'un règlement juridictionnel des différends auprès des États qui, tout en mesurant les contraintes afférentes à un tel mode de règlement des différends, voient cette contrainte contrebalancée par les avantages qu'ils retirent de leur participation à un ensemble conventionnel leur procurant des avantages supérieurs à ces contraintes. Le cadre de l'OMC est notamment particulièrement représentatif de cette progression Dès lors, la procéduralisation amène non seulement la garantie que des limites ne seront pas dépassées,

14 Voir notre étude : J. M. Sorel, Les multiples lectures d'un arrêt : entre sentiment d'impunité et sentiment de cohérence, une décision à relativiser, 2 RGDIP (2007), 259, 259 - 272 à propos de l'arrêt de la CIJ du 26 février 2007 dans l'affaire dite du Génocide entre la Bosnie-Herzégovine et l'ex-Yougoslavie.

15 Ce que les juges Tomka ou Stotnikov pointaient, tout comme le juge ad hoc Mahiou le suggéraient, en remarquant que la Cour n'aurait pas pu juger sans l'appui des preuves du TPIY.

16 Ruiz Fabri \& Sorel, supra note 5. 
mais apparaît surtout comme un moindre mal dans le bilan coût-avantages qu'ils peuvent opérer.

Mais surtout, cette liaison procéduralisation-légitimation ressort d'une tendance lourde de la société internationale, comme des ordres internes : celle de la légitimation par le droit, ou ce qui semble en être la représentation, alors que cette légitimation devrait être avant tout politique ou sociale, c'est-à-dire " extra-juridique ». On confond ainsi le lieu (le droit) où s'exprime cette légitimation comme résultat avec ses fondements ou son vecteur. Le droit devient légitimation via la procédure alors qu'il ne devrait en être que le réceptacle ou le vecteur. On inverse ainsi l'évolution naturelle du cadre juridique : créer un carcan donne l'illusion d'un bon droit, alors que le bon droit devrait être la résultante de ce carcan. Comme, au surplus, la procédure est technique, la propension à la scientificité (ou du moins à la technicité) est aux portes de cette procéduralisation.

Celui qui consent - que ce soit dans un processus facultatif ou dans un processus obligatoire résultant d'un cadre conventionnel - paraît dépassé par le consentement, surtout à partir du moment où il n'est plus le seul justiciable. Il lui faut donc une bouée, et la procédure représente pour lui cette bouée. Il en ressort cette impression que la légitimation passe par le respect des formes, alors qu'elle devrait passer par la confiance faite en la juridiction, ce qui ne veut pas dire pour autant qu'il faut négliger la procédure. Cela nous amène pour conclure à la question de l'idée de justice qui émerge de ce processus.

\section{La transformation progressive de l'idée de justice à travers la procéduralisation}

La procéduralisation semble être une tendance qui n'est pas isolée et qui gagne tout le droit international en dehors des juridictions. Ce caractère correctif ou invasif des règles de procédure apparait comme une tendance générale du droit international. Paradoxalement, on peut s'interroger sur la perte de confiance dans le droit à son origine, et sur la perte de confiance dans la justice pour le domaine qui nous intéresse. On ressent en effet une forme de transformation progressive de l'idée de justice à travers la procéduralisation.

La transparence, les audiences publiques, l'amicus curiae, la place de l'indépendance et l'impartialité, le rôle des experts, le rôle de la preuve, etc., sont autant d'exemples de l'importance de la procéduralisation en droit international. Si les progrès sont indéniables (et loin de nous l'idée de ne voir ce phénomène que négativement), cela semble aussi révéler un malaise 
plus profond. En effet, paradoxalement, cette procéduralisation marque aussi une perte de confiance dans la justice.

La procéduralisation est la marque d'un droit international qui se modifie et quelque part se banalise et remet en cause son identité qui ne s'assimile ni à son statut, ni à son exorbitance publique, mais surtout à sa nécessité. Bien sûr, il y a un droit statutaire, bien sûr il existe un droit institutionnel, bien sûr il y a des exorbitances, mais comme dans cette matière on privilégie les relations horizontales, rien n'est évident, tout doit être construit et il y a peu de donné. C'est un droit fondamentalement fonctionnel, audelà de l'institutionnel ou du matériel. ${ }^{17}$ En simplifiant et en généralisant, on part d'une situation et on essaie de lui trouver une solution, mais on ne part pas d'un état statutaire auquel s'adapterait la solution hiérarchisée, d'où découlerait une solution qui ruissellerait ensuite sur la pyramide.

Dès lors, si modèle procédural il y a, celui-ci doit être original et ne pas se fier à ce qui existe dans un État. Mais surtout, il faut remarquer que la justice est apparue d'une certaine manière dans l'histoire de l'État : elle a émané d'une démarche de séparation des pouvoirs et pour en garantir la stabilité. A l'inverse, en droit international, la justice est apparue comme un élément tiers qu'on mettait à côté, une sorte d'excroissance, que les États ont progressivement acceptée. La transposition de la traditionnelle séparation des pouvoirs ne sert donc pas à grand-chose dans ce cadre, ${ }^{18}$ même si l'idée qu'il doit exister des juridictions internationales auxquelles les Etats et les individus se soumettent est entrée dans les mœurs. ${ }^{19}$

Mais, alors même que la greffe est difficile, les juridictions internationales, sous l'effet de la consolidation de la justice internationale pénale, reflètent une tendance qui s'accentue en droit interne : la quête éperdue de la responsabilisation qui inhibe les sociétés modernes. Dans le domaine pénal, la chasse aux responsables a tendance à remplacer la quête des faits ou la quête de la vérité. En un mot : il arrive que le juste prime le vrai parce que le discours politique impose logiquement un devoir de mémoire, mais qu'il double ce devoir d'exigences en termes de responsabilité et de réparations, exigences pas toujours compatibles avec la complexe réalité du

17 Voir nos remarques dans : J. M. Sorel, L'identité du droit international ou la posture du sans papiers heureux, in X. Bioy (dir.), L'identité du droit public (2011), $147-158$.

18 Voir nos remarques dans : J. M. Sorel, Les Tribunaux pénaux internationaux : ombre et lumière d'une récente grande ambition, 1(205) Revue Tiers Monde (2011), 29.

19 Voir nos remarques dans : J. M. Sorel, Les juridictions internationales : un ensemble hétérogène et partiel, 49 Questions internationales (2011), 77. 
conflit. ${ }^{20}$ Dans ce cadre, la procédure tient une place centrale pour isoler la responsabilité, sans qu'il soit certain que cette même procédure permette d'embrasser la complexité de la vérité des faits.

Le procès international semble ainsi, via la procéduralisation, subir une forme d'auto-poïèse. Il s'auto-entretient et renforcer l'échafaudage de la procédure devient une forme d'architecture finale en elle-même. En un mot, le respect de la procédure s'impose parfois plus rapidement que l'idée de justice elle-même et, en droit international, plus rapidement que les juridictions elles-mêmes. Or, sans la jeter en pâture aux tenants d'une justice expéditive et dépourvue de toute garantie, on est en droit de se demander si cette forme de procéduralisation n'est pas une atteinte au caractère fondamentalement fonctionnaliste du droit international. Simple interrogation. Toute réponse péremptoire est à bannir.

20 Ruiz Fabri \& Sorel, supra note 5. 
